\title{
THALIDOMIDE: A THERAPEUTIC BOON IN THE MANAGEMENT OF RECURRENT GENITAL APHTHOUS ULCERATION IN AN IMMUNOCOMPROMISED INDIVIDUAL
}

\author{
Ch. Madhubabu', P. Padmaja², T. Rajeev Singh ${ }^{3}$, Gautham Krishna Reddy4, Sridhar Thatipally5, Todeti Yashwanth ${ }^{6}$
}

${ }_{1}^{1}$ Professor, Department of Dermatology, Venereology and Leprosy, Osmania Medical College.

${ }^{2}$ Associate Professor, Department of Dermatology, Venereology and Leprosy, Osmania Medical College.

${ }^{3}$ Assistant Professor, Department of Dermatology, Venereology and Leprosy, Osmania Medical College.

4 Junior Resident, Department of Dermatology, Venereology and Leprosy, Osmania Medical College.

5 Junior Resident, Department of Dermatology, Venereology and Leprosy, Osmania Medical College.

6Junior Resident, Department of Dermatology, Venereology and Leprosy, Osmania Medical College.

\begin{abstract}
Genital lesions are common in Human Immunodeficiency Virus (HIV) positive patients. Aetiology of the genital lesions are mainly due to Herpes Simplex Virus (HSV), Human Papilloma Virus (HPV) and non-specific genital ulcers. They usually respond to the antiviral and antibacterial agents. We are presenting a young unmarried HIV positive and Hepatitis-B positive patient who presented with non-healing genital ulcer lesions while on antiretroviral therapy and showed variable response and challenges to treatment with antivirals, steroids, antibacterials and change of ART and finally dramatic response with Thalidomide therapy which is rarely described in the literature.
\end{abstract}

\section{KEYWORDS}

Genital Ulcers, HIV, Hepatitis-B, Thalidomide.

HOW TO CITE THIS ARTICLE: Madhubabu Ch, Padmaja P, Singh TR, et al. Thalidomide: a therapeutic boon in the management of recurrent genital aphthous ulceration in an immunocompromised individual. J. Evolution Med. Dent. Sci. 2016;5(52):3468-3470, DOI: $10.14260 /$ jemds/2016/800

\section{INTRODUCTION}

Genital lesions are common in HIV positive patients.(1) Aetiology of the genital lesions are mainly due to Herpes Simplex Virus (HSV), Human Papilloma Virus (HPV) and nonspecific genital ulcers.(2) They usually respond to the antiviral and antibacterial agents.

\section{CASE REPORT}

Thirty year old unmarried male, known positive for HIV infection and Hepatitis-B infection, on Highly Active Antiretroviral Therapy (HAART) since 2007, presented with genital ulcers for a prolonged duration of 22 months. Initially he developed small fluid filled lesions over inner surface of prepuce, which ruptured to form erosions and they slowly progressed to involve glans associated with pain. No other associated systemic symptoms. Outside biopsy was done which showed non-specific inflammatory dermatitis and he was treated in the lines of genital herpes infection by private practitioners with tab Acyclovir $800 \mathrm{mg}$ tid for 1 month; Famciclovir 500 mg twice daily for 1 wk.; Tab Azithromycin 1 gm stat; Tab Cefixime 400 mg od for 1 wk.; Tab Ceftriaxone 1 gm BD for 2 wks. Past history of exposure 13 yrs. back, 3-4 times, with unknown person, unprotected. History of similar complaints in past on and off for every 3-5 months from last 13 yrs., initially took acyclovir $200 \mathrm{mg}$ tid, later $400 \mathrm{mg}$ tid and then $800 \mathrm{mg}$ tid; showed a variable response; was diagnosed as RVD 12 yrs. back but started ART 7 yrs. back, initially Tab Vonaday (Tenofovir $300 \mathrm{mg}$, Lamivudine 300 $\mathrm{mg}$, Efavirenz $600 \mathrm{mg}$ ).

Financial or Other, Competing Interest: None.

Submission 20-04-2016, Peer Review 08-06-2016,

Acceptance 13-06-2016, Published 30-06-2016.

Corresponding Author:

Dr. Ch. Madhubabu,

Flat No. 202, Lakshmi Nilayam,

RTC Colony, Champapet,

Hyderabad-500079, Telangana.

E-mail: drchmadhubabu@gmail.com

DOI: $10.14260 /$ jemds/2016/800
In 2014, was shifted to Tab Forstavir-3 (Efavirenz 600 $\mathrm{mg}$, Emtricitabine $200 \mathrm{mg}$, Tenofovir $300 \mathrm{mg}$ ). On examination, multiple small round non-indurated painful ulcers over glans and mucosal part of prepuce with red granulation tissue covered by yellowish slough and necrotic material (Figure 1a); Bleeding on touch with bilateral inguinal lymphadenopathy present which are discrete, nontender, 3 on each side measuring $1.5^{*} 2 \mathrm{~cm}$ without any urethral discharge. Testis and scrotal contents were normal. Oral and anal mucosa were normal. Skin, hair and nails were normal.

Investigations: CBP/ESR/CUE/RFT/LFT/RBS were within normal limits. HIV-I: Reactive, HBsAg: Reactive; CD4: 385.08 cells/cmm; CD8: 934.4 cells $/ \mathrm{cmm} ;$ CD4:CD8 Ratio $=0.41$; Ultrasound Abdomen: no organomegaly; HSV IgG Antibodies: 3.33 (Significant $>1.5$ ); Tzank smear: negative; Tissue smear for grams stain: no bacteria; VDRL: non-reactive; TPHA: negative; Mantoux test: negative; Blood for TB PCR: negative; Biopsy: Aphthous ulcer.

As biopsy report came as Aphthous ulceration, pt. was started on Tab Prednisolone $20 \mathrm{mg}$ bd for a wk., later tapered to $20 \mathrm{mg}$ morning and $10 \mathrm{mg}$ night for next wk., $10 \mathrm{mg}$ BD for next 2 wks. As there was no response, drug was tapered over a period of 8-10 wks.

Thinking in terms of Persistent Herpes Genitalis, pt. was again put on Tab Valacyclovir $1 \mathrm{gm}$ BD for 2 wks. Also ART was stopped simultaneously, thinking ART to be the cause of genital ulcerations. But there was no response.

Later thinking of fusospirochetes infection, Tab. Dapsone $100 \mathrm{mg}$ OD, Tab. Prednisolone $20 \mathrm{mg}$ BD for 3 days; $20 \mathrm{mg}$ morning, $10 \mathrm{mg}$ night for next 3 days and $10 \mathrm{mg}$ BD for remaining days was continued for a total of 2 wks., but no response was found.

Later, all medications were stopped and therapeutic trial was started for Syphilis. Injection Benzathine Penicillin 24 lakh units with 12 lakh units in each buttock, deep intramuscular injection was given. After a single dose, ulcers started to heal and second and third dose of injection 
Benzathine Penicillin was given a week apart. Ulcers almost healed (Figure-1b, 1c, 1d), but after a period 2 months later patient again presented with a small erosion, which slowly progressed to ulcerations in due course forming large exuberant ulceration involving glans penis, prepuce and encroaching onto the urethral meatus with purulent subpreputial discharge (Figure-1e).

Later thought of changing ART in view of ART induced drug ulcerations, which are documented in literature and we kept patient on combination of ART drugs containing 2 NRTIs containing Tab. Tenofovir $300 \mathrm{mg}$ once daily, Tab Lamivudine $300 \mathrm{mg}$ once daily with 1 Boosted PI containing Tab Lopinavir $200 \mathrm{mg}$ with Tab Ritonavir $50 \mathrm{mg}$ given in a dose of 2 tab twice daily. On follow-up, there was no clinical response for the above combination even after 6-8 weeks of initiation of therapy (Figure-1f).

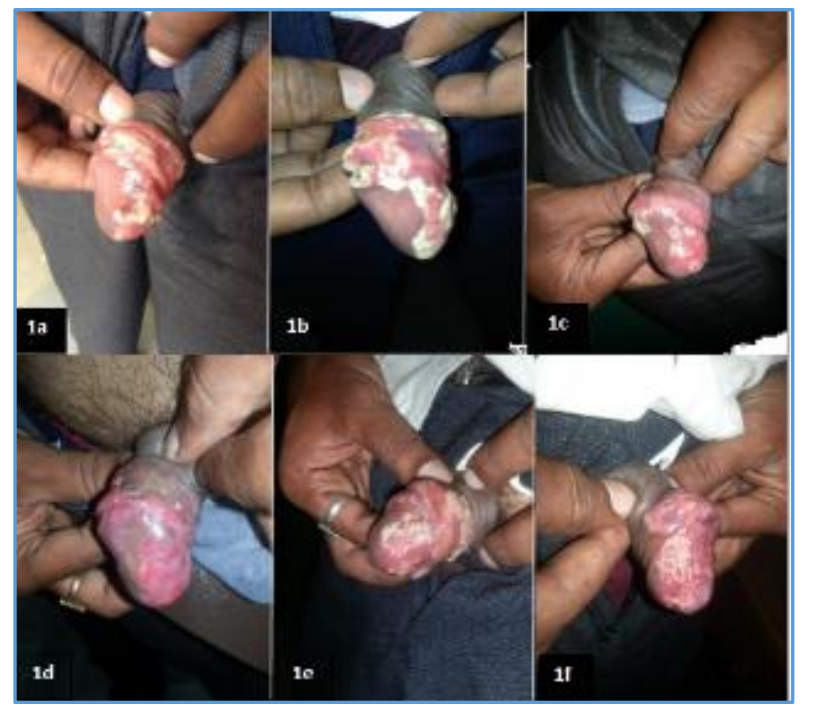

Fig. 1: 1a-Genital Ulcer at the Time of Presentation to Our Hospital ; 1 b-Before Starting Inj. Benzathine Penicillin ; $1 c$ One Week After $1^{\text {st }}$ Dose of Inj. Benzathine Penicillin ; 1d-3 Weeks After Third Dose of Inj. Benzathine Penicillin ; 1 eTwo Months After Third Dose of Inj. Benzathine Penicillin; 1f-One Week After Changing ART Regimen

Repeat biopsy was done, which showed possibility of Aphthous ulceration with features of foci of ulceration with necrotic area and neutrophilic infiltrate in epidermis and extensive perivascular infiltrate of lymphocytes, histiocytes and few neutrophils in dermis. Thinking in terms of Recalcitrant Aphthous ulcerations in an immunocompromised individual having a combined infection of HIV and Hepatitis B not responding to any of the modalities indicated for genital aphthous ulcerations and after review of literature, patient was started on Tab. Thalidomide $100 \mathrm{mg}$ thrice daily on $5^{\text {th }}$ December 2015 (Figure-2a, 2b). Within a week, ulcers started to heal slowly and after a period of 3-4 weeks a good response was obtained (Figure-2c). Tab. Zinc was added as a supplement. By the end of 8 weeks, there was complete resolution of ulcer without evidence of any scar formation with a normal genital architecture (Figure-2d) and we continued Thalidomide therapy for another 4 weeks, completing a total therapy of 12 weeks (Figure-2e, 2f).

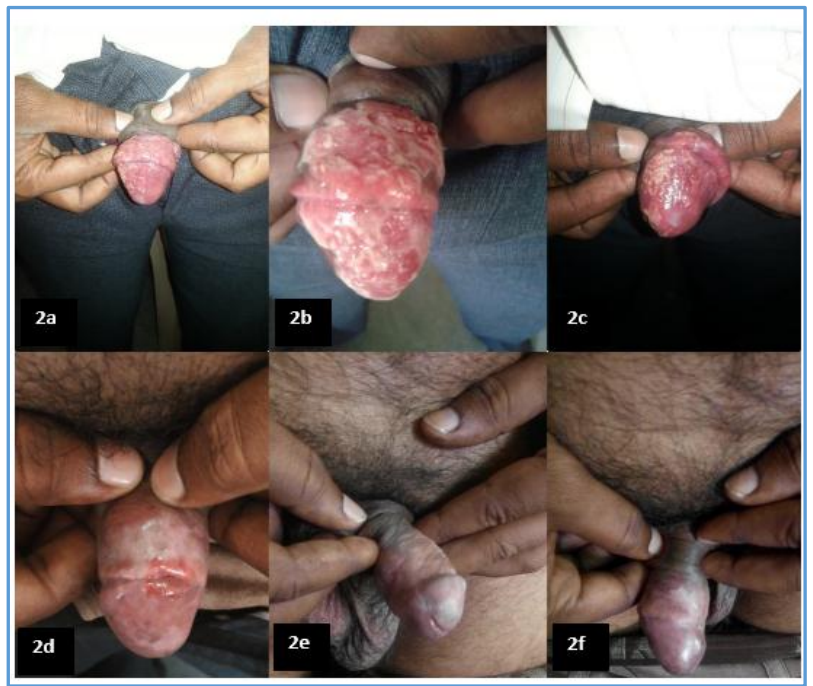

Fig. 2: $2 a$ \& 2b-Before Starting Tab. Thalidomide; $2 c$ - Three Weeks After Starting Tab. Thalidomide; 2d-8 Weeks After Starting Tab. Thalidomide; 2 e \& $2 f-12$ Weeks After Completing Tab. Thalidomide Therapy

\section{DISCUSSION}

Genital ulcerations in HIV positive patients can be of infectious or non-infectious causes. Most common being infectious causes, that too sexually transmitted diseases among which Herpes Simplex Virus type 2 (HSV-2) is the dominant primary causative agent in genital ulcerative infections in HIV positive patients. Other infectious causes being Chancroid, Syphilis, etc. Non-infectious causes are Aphthous ulcerations, Traumatic ulcerations, Malignancies, etc. (3)

Prevalence of Genital Ulcer Disease (GUD) is high in highrisk HIV-positive patients and increases with declining immunity.(1) In our patient, he gave past history of herpes and he was improving with acyclovir previously.

Genital herpes was the main aetiology of Chronic Genital Ulcer (CGU) in patients with HIV infection. Conversely, chancroid was the main aetiology in patients without HIV infection. This finding suggests that herpetic CGU is highly associated with HIV Infection than to Chancroid CGU.

Our case is HIV and HBsAg positive on treatment with specific Antiviral therapy for both conditions, presenting with recurrent genital ulcerations for many years initially responding to Antiviral drugs showing HSV may be the cause. But later, our patient presented with a chronic non-healing ulcerations of prolonged duration, which did not respond to Antiviral drugs. Though biopsy showed Aphthous ulceration, there was no response to corticosteroids. This may be due to immunosuppression and co-infection of HIV and HBsAg.

There was a better response to Inj. Benzathine Penicillin compared to Antivirals drugs and Corticosteroids, which may be due to polymicrobial organisms implicated in aetiopathogenesis of genital ulcerations in HIV and Hepatitis $\mathrm{B}$ infected individuals, which may dramatically respond to therapy with Penicillins and its derivatives. Though the response lasted only for a few weeks. (4)

There were articles which showed atypical genital HSV lesions occurring in HIV/AIDS patients and change in HAART with boosted Atazanavir-containing PI regimen was associated with healing of chronic HSV genital lesions in an 
HIV/AIDS patient with improvement of CD4 count and immune reconstitution.

Whether Atazanavir has any direct role in healing of genital ulcers needs further investigation. Based on this, in our patient we changed ART to 2NRTIs containing Tab. Tenofovir $300 \mathrm{mg}$ once daily, Tab. Lamivudine $300 \mathrm{mg}$ once daily with 1 Boosted PI containing Tab. Lopinavir $200 \mathrm{mg}$ with Tab. Ritonavir $50 \mathrm{mg}$ given in a dose of 2 tabs twice daily, but there was no improvement.

Based on multiple literature reviews in such type of presentations, the option of Thalidomide was considered.(5) Patient was started on Thalidomide therapy in a dose of 100 $\mathrm{mg}$ three times daily for a period of 12 weeks. Within a week of initiation of treatment, dramatic response was noted in the healing of ulcer with gradual improvement in the clinical presentation over the next few weeks.

By the end of 8 weeks, complete resolution of the ulceration was found with restoration of the normal genital architecture. Patient was continued on Thalidomide therapy $100 \mathrm{mg}$ three times daily for another 4 weeks, thus completing a total treatment of 12 weeks. Patient was regularly followed up and screened for all the side effects of Thalidomide based on the monitoring guidelines. Patient never manifested any drug complications during the therapy.

Patient should be continued on ART therapy with all above mentioned drugs and the drug Tenofovir was selected in view of its dual action on both HIV and Hepatitis-B infection and showing a promising therapeutic effect, especially on Hepatitis-B infection. Tenofovir being the main drug combating the Hepatitis-B infection in recent years.

All the drugs which were beneficial in the treatment of immunocompetent individuals with genital aphthous ulcerations did not show any promising results in our case of dual infection of HIV and Hepatitis-B. As a last option, we considered the drug Thalidomide in this case which gave unbelievable dramatic and persistent results throwing a therapeutic challenge to the clinicians and finally giving a complete clinical cure to the patient allaying all his psychological components of nearly 2 years duration and to become an active member in the society.

\section{CONCLUSION}

This case has been presented for its rarity of co-infection of HIV and Hepatitis-B infection in an immunocompromised individual presenting with persistent genital ulcerations of prolonged duration creating a lot of dilemma in clinical, investigative and therapeutic evaluation with the patient being put to multiple modalities of treatment with brief period of remissions and again with subsequent recurrences causing lot of psychological trauma, both to the clinician and patient which could finally be balanced and stabilised with complete cure and resolution with the drug Thalidomide in a complete therapeutic dosages to the point of complete clinical response. So, in such above type of cases the drug Thalidomide can be considered as a best therapeutic option and promising agent with proper screening of toxicities during the course of treatment.

\section{REFERENCES}

1. Ghys PD, Diallo MO, Traore-Ettiegne V, et al. GUD prevalence is high in HIV-positive prostitutes and increases with declining immunity. Int Conf AIDS 1993;9:660.

2. Gbery IP, Djeha D, Kacou DE, et al. Chronic genital ulcerations and HIV infection: 29 cases. Med Trop (Mars) 1999;59(3):279-82.

3. Augenbraun MH. Diseases of the reproductive organs and sexually transmitted diseases: genital skin and mucous membrane lesions. In: Mandell GL, Bennett JE, Dolin R, eds. Mandell, Douglas, and Bennett's principles and practice of infectious diseases. $7^{\text {th }}$ ed. Philadelphia, Pa. Churchill Livingstone 2009:1475-84.

4. Kumar SA, Kumar N, Kumarasamy N. Genital lesions: an indication for changing ART regimen. Indian Journal of Sexually Transmitted Diseases 2011;32(1):37-9. doi:10.4103/0253-7184.81253.

5. Radeff B, Kuffer R, Samson J. Recurrent aphthous ulcer in patient infected with human immunodeficiency virus: successful treatment with thalidomide. J Am Acad Dermatol 1990;23:523-5. 